Acta Crystallographica Section E

Structure Reports

Online

ISSN 1600-5368

\section{catena-Poly[[diaquacadmium(II)]- $\mu-4,4^{\prime}$ - sulfonyldibenzoato- $\left.\kappa^{2} O^{1}: O^{1}\right]$}

\section{Chang-mei Jiao}

Department of Chemistry, Yancheng Teachers' College, Yancheng 224002, People's Republic of China

Correspondence e-mail: wjndyc@gmail.com

Received 25 September 2010; accepted 4 October 2010

Key indicators: single-crystal X-ray study; $T=298 \mathrm{~K}$; mean $\sigma(\mathrm{C}-\mathrm{C})=0.002 \AA$; $R$ factor $=0.032 ; w R$ factor $=0.102 ;$ data-to-parameter ratio $=12.4$.

The title compound, $\left[\mathrm{Cd}\left(\mathrm{C}_{14} \mathrm{H}_{8} \mathrm{O}_{6} \mathrm{~S}\right)\left(\mathrm{H}_{2} \mathrm{O}\right)_{2}\right]_{n}$, comprises zigzag chains parallel to [111] of alternating $\left[\mathrm{Cd}\left(\mathrm{H}_{2} \mathrm{O}\right)_{2}\right]^{2+}$ and sulfonyldibenzoate units, with the $\mathrm{Cd}$ and $\mathrm{S}$ atoms lying on crystallographic twofold axes. The central $\mathrm{Cd}^{\mathrm{II}}$ ion is in a slightly distorted octahedral geometry, coordinated by six $\mathrm{O}$ atoms from two carboxylate groups and two water $\mathrm{O}$ atoms. An intramolecular $\mathrm{C}-\mathrm{H} \cdots \mathrm{O}$ hydrogen bond occurs. In the crystal, intermolecular hydrogen bonds between carboxylate $\mathrm{O}$ atoms and coordinated water molecules in adjacent chains lead to the formation of a three-dimensional network structure. The compound is isotypic with the $\mathrm{Zn}$ analog.

\section{Related literature}

For related compounds based on 4,4'-sulfonyldibenzoic acid, see: Xiao et al. (2007); Wu et al. (2007); Miyazawa et al. (2009); Wang et al. (2009). For the isotypic $\mathrm{Zn}$ analog, see: Pan et al. (2007). For potential application of metal-organic frameworks, see: Eddaoudi et al. (2001); Ferey et al. (2005); Kitagawa et al. (2004).

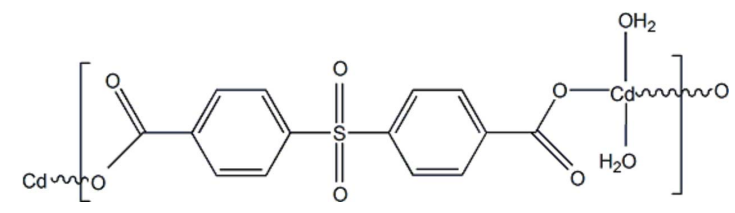

\section{Experimental}

Crystal data

$\left[\mathrm{Cd}\left(\mathrm{C}_{14} \mathrm{H}_{8} \mathrm{O}_{6} \mathrm{~S}\right)\left(\mathrm{H}_{2} \mathrm{O}\right)_{2}\right]$

$M_{r}=452.72$

Monoclinic, $P 2 / c$

$$
\begin{aligned}
& a=13.293(3) \AA \\
& b=5.2742(12) \AA \\
& c=12.156(3) \AA
\end{aligned}
$$

$\beta=116.145(2)^{\circ}$

$V=765.1(3) \AA^{3}$

$Z=2$

Mo $K \alpha$ radiation

$\mu=1.61 \mathrm{~mm}^{-1}$

$T=298 \mathrm{~K}$

$0.21 \times 0.19 \times 0.15 \mathrm{~mm}$

Data collection

Bruker SMART CCD area-detector diffractometer

Absorption correction: multi-scan (SADABS; Bruker, 2000)

$T_{\min }=0.721, T_{\max }=0.786$

3574 measured reflections 1364 independent reflections 1325 reflections with $I>2 \sigma(I)$ $R_{\text {int }}=0.073$

Refinement

$R\left[F^{2}>2 \sigma\left(F^{2}\right)\right]=0.032$

$w R\left(F^{2}\right)=0.102$

$S=1.24$

1361 reflections

110 parameters

3 restraints

$\mathrm{H}$-atom parameters constrained

$\Delta \rho_{\max }=0.82{\mathrm{e} \AA^{-3}}^{-3}$

$\Delta \rho_{\min }=-1.00 \mathrm{e}^{-3}$

Table 1

Hydrogen-bond geometry $\left(\AA{ }^{\circ}\right)$.

\begin{tabular}{lllll}
\hline$D-\mathrm{H} \cdots A$ & $D-\mathrm{H}$ & $\mathrm{H} \cdots A$ & $D \cdots A$ & $D-\mathrm{H} \cdots A$ \\
\hline $\mathrm{O} 4-\mathrm{H} 4 B \cdots \mathrm{O} 1^{\mathrm{i}}$ & 0.85 & 1.97 & $2.7479(14)$ & 151 \\
$\mathrm{O}^{\mathrm{i}}-\mathrm{H} 4 A \cdots \mathrm{O} 2^{\mathrm{ii}}$ & 0.85 & 2.00 & $2.7364(15)$ & 145 \\
$\mathrm{C} 6-\mathrm{H} 6 \cdots \mathrm{O} 3$ & 0.93 & 2.55 & $2.9208(16)$ & 104 \\
\hline
\end{tabular}

Symmetry codes: (i) $x, y-1, z$; (ii) $x,-y, z-\frac{1}{2}$.

Data collection: SMART (Bruker, 2000); cell refinement: SAINT (Bruker, 2000); data reduction: $S A I N T$; program(s) used to solve structure: SHELXS97 (Sheldrick, 2008); program(s) used to refine structure: SHELXL97 (Sheldrick, 2008); molecular graphics: SHELXTL (Sheldrick, 2008) and DIAMOND (Brandenburg, 1999); software used to prepare material for publication: SHELXTL.

Supplementary data and figures for this paper are available from the IUCr electronic archives (Reference: BX2311).

\title{
References
}

Brandenburg, K. (1999). DIAMOND. Crystal Impact GbR, Bonn, Germany. Bruker (2000). SMART, SAINT and SADABS. Bruker AXS Inc., Madison, Wisconsin, USA.

Eddaoudi, M., Moler, D. B., Li, H., Chen, B., Reineke, T. M., O'Keeffe, M. \& Yaghi, O. M. (2001). Acc. Chem. Res. 34, 319-330.

Ferey, G., Mellot-Draznieks, C., Serre, C. \& Millange, F. (2005). Acc. Chem. Res. 38, 217-225.

Kitagawa, S., Kitaura, R. \& Noro, S. (2004). Angew. Chem. Int. Ed. 43, 23342375.

Miyazawa, M., Irie, Y., Kashimoto, K., Nishina, N., Kondo, M., Yasue, S., Maeda, K. \& Uchida, F. (2009). Inorg. Chem. Commun. 12, 336-339.

Pan, P.-B., Zhang, L., Li, Z.-J., Cao, X.-Y. \& Yao, Y.-G. (2007). Acta Cryst. C63, $\mathrm{m} 270-\mathrm{m} 272$.

Sheldrick, G. M. (2008). Acta Cryst. A64, 112-122.

Wang, C.-J., Wang, Y.-Y., Liu, J.-Q., Wang, H., Shi, Q.-Z. \& Peng, S.-M. (2009). Inorg. Chim. Acta, 362, 543-550.

Wu, H.-H., Lian, F.-Y., Yuan, D.-Q. \& Hong, M.-C. (2007). Acta Cryst. E63, m67-m69.

Xiao, D.-R., Li, Y.-G., Wang, E.-B., Fan, L.-L., An, H.-Y., Su, Z.-M. \& Xu, L. (2007). Inorg. Chem. 46, 4158-4166. 


\section{supporting information}

Acta Cryst. (2010). E66, m1405 [https://doi.org/10.1107/S1600536810039711]

\section{catena-Poly[[diaquacadmium(II)]- $\mu$-4, $4^{\prime}$-sulfonyldibenzoato- $\kappa^{2} O^{1}: O^{1}$ ]}

\section{Chang-mei Jiao}

\section{S1. Comment}

In recent years, much attention has been focused on the construction of metal organic frameworks (MOFs) not only because of their fascinating structures and topologies but also owing to their potential application in many fields such as magnetism, catalysis, nonlinear optics. (Eddaoudi, et al., 2001; Kitagawa et al., 2004; Ferey et al., 2005.). The main method to construct such complexes is to use multidentate organic ligands. The organic aromatic polycarboxylate ligands are an important family of multidentate ligands. The 4,4'-sulfonyldibenzoic acid has been widely used in the construction of metal organic frameworks because of two carboxylate functions and its structural flexibility.(Xiao et al., 2007; Wu et al., 2007; Miyazawa et al., 2009; Wang et al., 2009.) We report here the synthesis and crystal structure of the title compound (I) based on 4,4'-sulfonyldibenzoic acid which is isostructural to the reported compound by Pan et al., 2007.

As shown in Fig. 1, the Cd centres in (I) are six-coordinate in a highly distorted octahedral geometry, involving four $\mathrm{O}$ atom donors of two 4,4'-sulfonyldibenzoic acid ligands and two coordinated water molecules, while the carboxylate group of 4,4'-sulfonyldibenzoic acid adopts $\mu_{2}-\eta^{1}: \eta^{1}$ - chelating mode in this structure. The structure of (I) comprises zigzag chains of alternating $\left[\mathrm{Cd}\left(\mathrm{H}_{2} \mathrm{O}\right)_{2}\right]^{2+}$ and sulfonyldibenzoate unit, with their respective $\mathrm{Cd}$ and $\mathrm{S}$ atoms lying on crystallographic twofold axes. In the crystal structure there are three hydrogen bonds, two $\mathrm{O}-\mathrm{H} \cdots \mathrm{O}$ intermolecular and one $\mathrm{C}-\mathrm{H} \cdots \mathrm{O}$ intramolecular interactions, lead to the formation of a three dimensional network structure. Fig 2, Table 1.

\section{S2. Experimental}

The title compound, (I), was prepared by the hydrothermal reaction of $\mathrm{Cd}\left(\mathrm{NO}_{3}\right)_{2} \cdot 6 \mathrm{H}_{2} \mathrm{O}(34.5 \mathrm{mg}, 0.1 \mathrm{mmol}), 4,4$ 'sulfonyldibenzoic (30 mg, $0.1 \mathrm{mmol}), 1,4-\mathrm{Bis}(1,2,4$-triazol-1-yl)butane (19.2 mg, $0.1 \mathrm{mmol})$, and $\mathrm{NaOH}(8.0 \mathrm{mg}, 0.2$ mmol) in $\mathrm{H}_{2} \mathrm{O}(10 \mathrm{ml})$ was sealed in a $16 \mathrm{ml}$ Teflon-lined stainless steel container and heated at $180{ }^{\circ} \mathrm{C}$ for $72 \mathrm{~h}$. After cooling to room temperature, block colorless crystals of (I) were collected by filtration and washed by water and ethanol several times. (yield 47.25\%, based on Cd). Elemental analysis for $\mathrm{C}_{14} \mathrm{H}_{12} \mathrm{CdO}_{8} \mathrm{~S}(\mathrm{Mr}=452.72)$ : $\mathrm{C} 37.14, \mathrm{H} 2.67$; found: 43.61, H 2.69 .

\section{S3. Refinement}

$\mathrm{H}$ atoms bonded to coordinated water oxygen atom were located in a difference Fourier map and fixed in the refinement, with $U_{\text {iso }}(\mathrm{H})=1.2 U_{\text {eq }}(\mathrm{O})$. All C-bound $\mathrm{H}$ atoms were positioned in calculated positions and refined using a riding model, with $\mathrm{C}-\mathrm{H}=0.93$ ? (aromatic) and $U_{\text {iso }}(\mathrm{H})=1.2 U_{\mathrm{eq}}(\mathrm{C})$. 


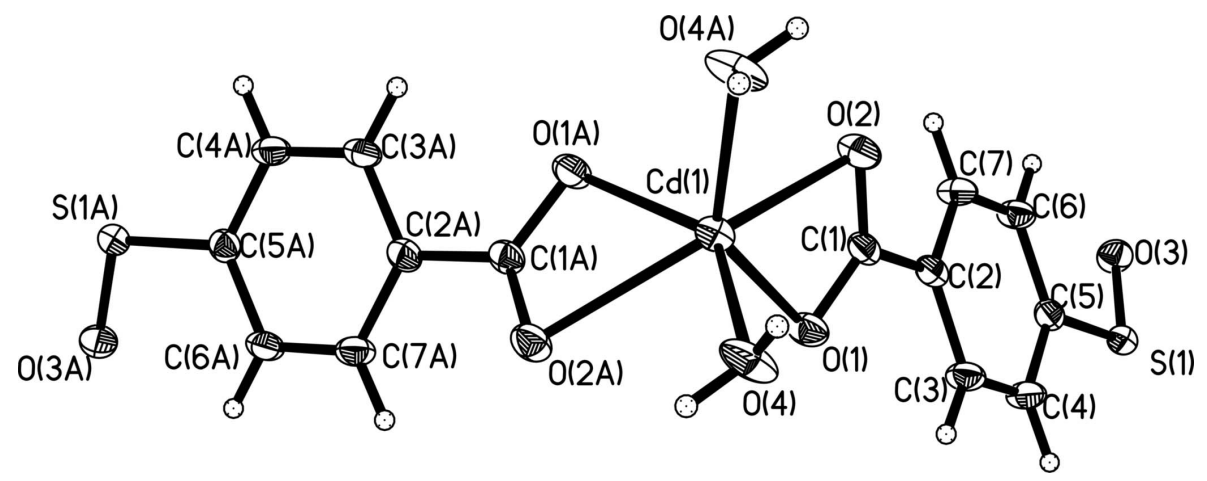

Figure 1

The structure of (I), showing the atom-numbering scheme. Displacement ellipsoids are plotted at the $30 \%$ probability level. [Symmetry codes: (A) $-\mathrm{x}, y, 3 / 2-z]$ 


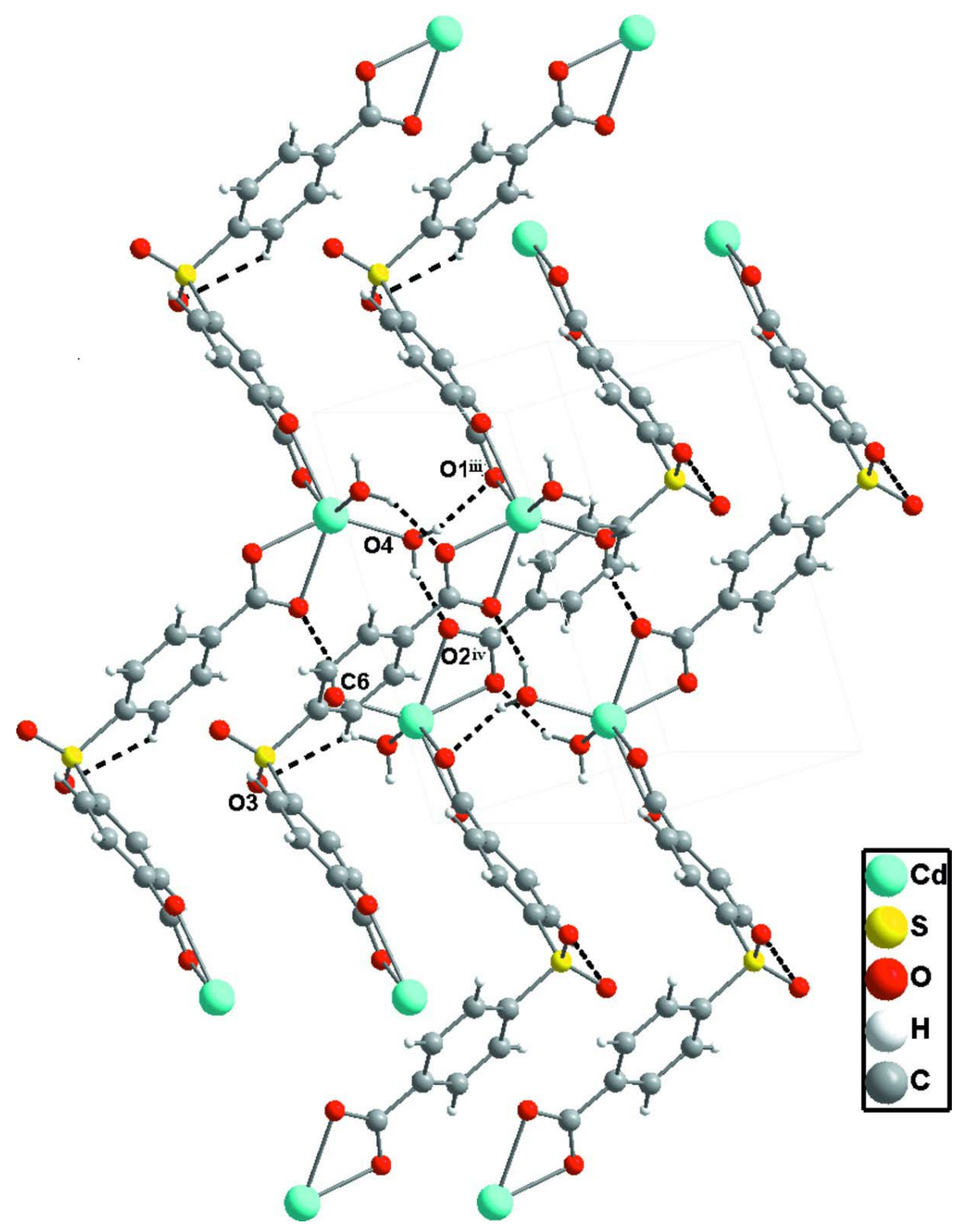

Figure 2

A view of (I), showing the three-dimensional framework constructed via $\mathrm{O}-\mathrm{H} \cdots \mathrm{O}$ hydrogen bonds. Hydrogen bonds are depicted as dashed lines. [Symmetry codes: (iii) $x, y-1, z$; (iv) $x,-y,-1 / 2+z$ ]

catena-Poly[[diaquacadmium(II)]- $\mu-4,4^{\prime}$-sulfonyldibenzoato- $\left.\kappa^{2} O^{1}: O^{1}\right]$

\section{Crystal data}

$\left[\mathrm{Cd}\left(\mathrm{C}_{14} \mathrm{H}_{8} \mathrm{O}_{6} \mathrm{~S}\right)\left(\mathrm{H}_{2} \mathrm{O}\right)_{2}\right]$

$M_{r}=452.72$

Monoclinic, $P 2 / c$

Hall symbol: -P $2 \mathrm{yc}$

$a=13.293(3) \AA$

$b=5.2742(12) \AA$

$c=12.156(3) \AA$

$\beta=116.145(2)^{\circ}$

$V=765.1(3) \AA^{3}$

$Z=2$
$F(000)=448$

$D_{\mathrm{x}}=1.965 \mathrm{Mg} \mathrm{m}^{-3}$

Mo $K \alpha$ radiation, $\lambda=0.71073 \AA$

Cell parameters from 4318 reflections

$\theta=2.2-27.2^{\circ}$

$\mu=1.61 \mathrm{~mm}^{-1}$

$T=298 \mathrm{~K}$

Block, white

$0.21 \times 0.19 \times 0.15 \mathrm{~mm}$ 


\section{Data collection}

Bruker SMART CCD area-detector diffractometer

Radiation source: fine-focus sealed tube Graphite monochromator

phi and $\omega$ scans

Absorption correction: multi-scan

(SADABS; Bruker, 2000)

$T_{\min }=0.721, T_{\max }=0.786$

\section{Refinement}

Refinement on $F^{2}$

Least-squares matrix: full

$R\left[F^{2}>2 \sigma\left(F^{2}\right)\right]=0.032$

$w R\left(F^{2}\right)=0.102$

$S=1.24$

1361 reflections

110 parameters

3 restraints

Primary atom site location: structure-invariant direct methods
3574 measured reflections

1364 independent reflections

1325 reflections with $I>2 \sigma(I)$

$R_{\text {int }}=0.073$

$\theta_{\max }=25.1^{\circ}, \theta_{\min }=1.7^{\circ}$

$h=-8 \rightarrow 15$

$k=-6 \rightarrow 6$

$l=-14 \rightarrow 12$

Secondary atom site location: difference Fourier map

Hydrogen site location: inferred from neighbouring sites

$\mathrm{H}$-atom parameters constrained

$w=1 /\left[\sigma^{2}\left(F_{\mathrm{o}}^{2}\right)+(0.0652 P)^{2}\right]$

where $P=\left(F_{\mathrm{o}}^{2}+2 F_{\mathrm{c}}{ }^{2}\right) / 3$

$(\Delta / \sigma)_{\max }=0.004$

$\Delta \rho_{\max }=0.82$ e $\AA^{-3}$

$\Delta \rho_{\text {min }}=-1.00$ e $\AA^{-3}$

Special details

Geometry. All e.s.d.'s (except the e.s.d. in the dihedral angle between two l.s. planes) are estimated using the full covariance matrix. The cell e.s.d.'s are taken into account individually in the estimation of e.s.d.'s in distances, angles and torsion angles; correlations between e.s.d.'s in cell parameters are only used when they are defined by crystal symmetry. An approximate (isotropic) treatment of cell e.s.d.'s is used for estimating e.s.d.'s involving 1.s. planes.

Refinement. Refinement of $F^{2}$ against ALL reflections. The weighted $R$-factor $w R$ and goodness of fit $S$ are based on $F^{2}$, conventional $R$-factors $R$ are based on $F$, with $F$ set to zero for negative $F^{2}$. The threshold expression of $F^{2}>\sigma\left(F^{2}\right)$ is used only for calculating $R$-factors(gt) $e t c$. and is not relevant to the choice of reflections for refinement. $R$-factors based on $F^{2}$ are statistically about twice as large as those based on $F$, and $R$ - factors based on ALL data will be even larger.

Fractional atomic coordinates and isotropic or equivalent isotropic displacement parameters $\left(\AA^{2}\right)$

\begin{tabular}{lllll}
\hline & $x$ & $y$ & $z$ & $U_{\text {iso }} * / U_{\text {eq }}$ \\
\hline Cd1 & 0.0000 & $0.06452(2)$ & 0.7500 & $0.03071(4)$ \\
S1 & 0.5000 & $1.11217(9)$ & 1.2500 & $0.02880(11)$ \\
O1 & $0.14145(7)$ & $0.34261(18)$ & $0.81726(7)$ & $0.0355(2)$ \\
O2 & $0.08657(7)$ & $0.25919(19)$ & $0.95927(7)$ & $0.0382(2)$ \\
O3 & $0.45660(6)$ & $1.24838(18)$ & $1.32215(7)$ & $0.0379(2)$ \\
O4 & $0.07556(8)$ & $-0.2236(2)$ & $0.67828(7)$ & $0.0504(3)$ \\
H4A & 0.0490 & -0.2211 & 0.6006 & $0.060^{*}$ \\
H4B & 0.0724 & -0.3725 & 0.7034 & $0.060^{*}$ \\
C1 & $0.14914(9)$ & $0.3776(3)$ & $0.92426(9)$ & $0.0286(2)$ \\
C2 & $0.23354(12)$ & $0.5638(2)$ & $1.00469(11)$ & $0.0304(4)$ \\
C3 & $0.32577(10)$ & $0.6180(3)$ & $0.98250(11)$ & $0.0354(3)$ \\
H3 & 0.3327 & 0.5418 & 0.9172 & $0.043 *$ \\
C4 & $0.40667(9)$ & $0.7851(3)$ & $1.05787(10)$ & $0.0365(3)$ \\
H4 & 0.4693 & 0.8180 & 1.0449 & $0.044^{*}$ \\
C5 & $0.39451(10)$ & $0.9035(2)$ & $1.15258(11)$ & $0.0290(3)$ \\
C6 & $0.30158(9)$ & $0.8548(3)$ & $1.17385(10)$ & $0.0351(3)$
\end{tabular}


supporting information

$\begin{array}{lllll}\text { H6 } & 0.2937 & 0.9353 & 1.2377 & 0.042 * \\ \text { C7 } & 0.22143(9) & 0.6857(3) & 1.09904(10) & 0.0349(3) \\ \text { H7 } & 0.1588 & 0.6533 & 1.1121 & 0.042^{*}\end{array}$

Atomic displacement parameters $\left(\AA^{2}\right)$

\begin{tabular}{lllllll}
\hline & $U^{11}$ & $U^{22}$ & $U^{33}$ & $U^{12}$ & $U^{13}$ & $U^{23}$ \\
\hline Cd1 & $0.03760(6)$ & $0.02817(8)$ & $0.02969(5)$ & 0.000 & $0.01787(4)$ & 0.000 \\
S1 & $0.03045(16)$ & $0.03051(18)$ & $0.02660(15)$ & 0.000 & $0.01361(12)$ & 0.000 \\
O1 & $0.0430(3)$ & $0.0402(4)$ & $0.0285(2)$ & $-0.0069(3)$ & $0.0206(2)$ & $-0.0069(3)$ \\
O2 & $0.0473(3)$ & $0.0419(5)$ & $0.0299(3)$ & $-0.0117(3)$ & $0.0211(2)$ & $-0.0022(3)$ \\
O3 & $0.0406(3)$ & $0.0381(5)$ & $0.0387(4)$ & $0.0037(4)$ & $0.0208(3)$ & $-0.0066(3)$ \\
O4 & $0.0902(5)$ & $0.0382(5)$ & $0.0411(3)$ & $0.0184(4)$ & $0.0456(3)$ & $0.0079(4)$ \\
C1 & $0.0348(4)$ & $0.0289(5)$ & $0.0234(3)$ & $0.0038(4)$ & $0.0140(2)$ & $0.0057(4)$ \\
C2 & $0.0345(5)$ & $0.0342(8)$ & $0.0240(5)$ & $0.0000(4)$ & $0.0143(4)$ & $0.0034(4)$ \\
C3 & $0.0375(5)$ & $0.0461(6)$ & $0.0309(4)$ & $-0.0039(5)$ & $0.0226(3)$ & $-0.0076(5)$ \\
C4 & $0.0348(4)$ & $0.0464(7)$ & $0.0378(4)$ & $-0.0055(5)$ & $0.0248(3)$ & $-0.0056(5)$ \\
C5 & $0.0305(5)$ & $0.0305(6)$ & $0.0253(5)$ & $0.0004(4)$ & $0.0116(4)$ & $0.0028(4)$ \\
C6 & $0.0394(5)$ & $0.0426(6)$ & $0.0314(4)$ & $-0.0039(6)$ & $0.0231(3)$ & $-0.0065(5)$ \\
C7 & $0.0375(4)$ & $0.0405(7)$ & $0.0364(4)$ & $-0.0060(5)$ & $0.0252(3)$ & $-0.0035(5)$ \\
& & & & & &
\end{tabular}

Geometric parameters $\left(\AA,{ }^{o}\right)$

\begin{tabular}{llll}
\hline $\mathrm{Cd} 1-\mathrm{O} 4$ & $2.2023(11)$ & $\mathrm{O} 4-\mathrm{H} 4 \mathrm{~A}$ & 0.8500 \\
$\mathrm{Cd} 1-\mathrm{O} 4^{\mathrm{i}}$ & $2.2023(11)$ & $\mathrm{O} 4-\mathrm{H} 4 \mathrm{~B}$ & 0.8499 \\
$\mathrm{Cd} 1-\mathrm{O} 1$ & $2.2362(10)$ & $\mathrm{C} 1-\mathrm{C} 2$ & $1.4871(16)$ \\
$\mathrm{Cd} 1-\mathrm{O} 1^{\mathrm{i}}$ & $2.2362(9)$ & $\mathrm{C} 2-\mathrm{C} 7$ & $1.385(2)$ \\
$\mathrm{Cd} 1-\mathrm{O} 2$ & $2.5040(9)$ & $\mathrm{C} 2-\mathrm{C} 3$ & $1.396(2)$ \\
$\mathrm{Cd} 1-\mathrm{O} 2^{\mathrm{i}}$ & $2.5040(10)$ & $\mathrm{C} 3-\mathrm{C} 4$ & 0.9300 \\
$\mathrm{Cd} 1-\mathrm{C} 1^{\mathrm{i}}$ & $2.7283(12)$ & $\mathrm{C} 3-\mathrm{H} 3$ & $1.380(2)$ \\
$\mathrm{S} 1-\mathrm{O} 3$ & $1.4367(10)$ & $\mathrm{C} 4-\mathrm{C} 5$ & 0.9300 \\
$\mathrm{~S} 1-\mathrm{O} 3^{\mathrm{ii}}$ & $1.4367(10)$ & $\mathrm{C} 4-\mathrm{H} 4$ & $1.393(2)$ \\
$\mathrm{S} 1-\mathrm{C} 5$ & $1.7655(12)$ & $\mathrm{C} 5-\mathrm{C} 6$ & $1.3798(17)$ \\
$\mathrm{S} 1-\mathrm{C} 5^{\mathrm{ii}}$ & $1.7655(12)$ & $\mathrm{C} 6-\mathrm{C} 7$ & 0.9300 \\
$\mathrm{O} 1-\mathrm{C} 1$ & $1.2725(15)$ & $\mathrm{C} 6-\mathrm{H} 6$ & 0.9300 \\
$\mathrm{O} 2-\mathrm{C} 1$ & $1.2550(17)$ & $\mathrm{C} 7-\mathrm{H} 7$ & $98.32(8)$ \\
& & & $86.34(7)$ \\
$\mathrm{O} 4-\mathrm{Cd} 1-\mathrm{O} 4^{\mathrm{i}}$ & $92.73(6)$ & $\mathrm{C} 1-\mathrm{O} 1-\mathrm{Cd} 1$ & 113.0 \\
$\mathrm{O} 4-\mathrm{Cd} 1-\mathrm{O} 1$ & $98.09(4)$ & $\mathrm{C} 1-\mathrm{O} 2-\mathrm{Cd} 1$ & 113.1 \\
$\mathrm{O} 4-\mathrm{Cd} 1-\mathrm{O} 1$ & $139.87(3)$ & $\mathrm{Cd} 1-\mathrm{O} 4-\mathrm{H} 4 \mathrm{~A}$ & 110.5 \\
$\mathrm{O} 4-\mathrm{Cd} 1-\mathrm{O} 1^{\mathrm{i}}$ & $139.87(3)$ & $\mathrm{Cd} 1-\mathrm{O} 4-\mathrm{H} 4 \mathrm{~B}$ & $120.51(10)$ \\
$\mathrm{O} 4-\mathrm{Cd} 1-\mathrm{O} 1^{\mathrm{i}}$ & $98.09(4)$ & $\mathrm{H} 4 \mathrm{~A}-\mathrm{O} 4-\mathrm{H} 4 \mathrm{~B}$ & $121.76(11)$ \\
$\mathrm{O} 1-\mathrm{Cd} 1-\mathrm{O} 1^{\mathrm{i}}$ & $98.02(5)$ & $\mathrm{O} 2-\mathrm{C} 1-\mathrm{O} 1$ & $117.73(12)$ \\
$\mathrm{O} 4-\mathrm{Cd} 1-\mathrm{O} 2$ & $126.84(3)$ & $\mathrm{O} 2-\mathrm{C} 1-\mathrm{C} 2$ & $119.83(11)$ \\
$\mathrm{O} 4-\mathrm{Cd} 1-\mathrm{O} 2$ & $88.07(4)$ & $\mathrm{O} 1-\mathrm{C} 1-\mathrm{C} 2$ & $121.45(14)$ \\
$\mathrm{O} 1-\mathrm{Cd} 1-\mathrm{O} 2$ & $54.80(3)$ & $\mathrm{C} 7-\mathrm{C} 2-\mathrm{C} 3$ & $118.72(13)$ \\
$\mathrm{O} 11^{\mathrm{i}}-\mathrm{Cd} 1-\mathrm{O} 2$ & $92.21(3)$ & $\mathrm{C} 7-\mathrm{C} 2-\mathrm{C} 1$ & $\mathrm{C} 3-\mathrm{C} 2-\mathrm{C} 1$ \\
$\mathrm{O} 4-\mathrm{Cd} 1-\mathrm{O} 2^{\mathrm{i}}$ & $88.07(4)$ & &
\end{tabular}




$$
\begin{aligned}
& \mathrm{O} 4-\mathrm{Cd} 1-\mathrm{O} 2^{\mathrm{i}} \\
& \mathrm{O} 1-\mathrm{Cd} 1-\mathrm{O} 2^{\mathrm{i}} \\
& \mathrm{O} 1^{\mathrm{i}}-\mathrm{Cd} 1-\mathrm{O} 2^{\mathrm{i}} \\
& \mathrm{O} 2-\mathrm{Cd} 1-\mathrm{O} 2^{\mathrm{i}} \\
& \mathrm{O} 4-\mathrm{Cd} 1-\mathrm{C}^{\mathrm{i}} \\
& \mathrm{O} 4-\mathrm{Cd} 1-\mathrm{Cl}^{\mathrm{i}} \\
& \mathrm{O} 1-\mathrm{Cd} 1-\mathrm{C}^{\mathrm{i}} \\
& \mathrm{O} 1^{\mathrm{i}}-\mathrm{Cd} 1-\mathrm{C}^{\mathrm{i}} \\
& \mathrm{O} 2-\mathrm{Cd} 1-\mathrm{Cl}^{\mathrm{i}} \\
& \mathrm{O} 2^{\mathrm{i}}-\mathrm{Cd} 1-\mathrm{C}^{\mathrm{i}} \\
& \mathrm{O} 3-\mathrm{S} 1-\mathrm{O}^{\mathrm{ii}} \\
& \mathrm{O} 3-\mathrm{S} 1-\mathrm{C} 5 \\
& \text { O3 } 3 \text { ii- } \mathrm{S} 1-\mathrm{C} 5 \\
& \mathrm{O} 3-\mathrm{S} 1-\mathrm{C} 5^{\mathrm{ii}} \\
& \mathrm{O} 3^{\mathrm{ii}}-\mathrm{S} 1-\mathrm{C} 5^{\mathrm{ii}} \\
& \mathrm{C} 5-\mathrm{S} 1-\mathrm{C} 5^{\mathrm{ii}} \\
& \mathrm{O} 4-\mathrm{Cd} 1-\mathrm{O} 1-\mathrm{C} 1 \\
& \mathrm{O} 4-\mathrm{Cd} 1-\mathrm{O} 1-\mathrm{C} 1 \\
& \mathrm{O} 1-\mathrm{Cd} 1-\mathrm{O} 1-\mathrm{C} 1 \\
& \mathrm{O} 2-\mathrm{Cd} 1-\mathrm{O} 1-\mathrm{C} 1 \\
& \mathrm{O} 2-\mathrm{Cd} 1-\mathrm{O} 1-\mathrm{C} 1 \\
& \mathrm{C} 1-\mathrm{Cd} 1-\mathrm{O} 1-\mathrm{C} 1 \\
& \mathrm{O} 4-\mathrm{Cd} 1-\mathrm{O} 2-\mathrm{C} 1 \\
& \mathrm{O} 4-\mathrm{Cd} 1-\mathrm{O} 2-\mathrm{C} 1 \\
& \mathrm{O} 1-\mathrm{Cd} 1-\mathrm{O} 2-\mathrm{C} 1 \\
& \mathrm{O} 1-\mathrm{Cd} 1-\mathrm{O} 2-\mathrm{C} 1 \\
& \mathrm{O} 2-\mathrm{Cd} 1-\mathrm{O} 2-\mathrm{C} 1 \\
& \mathrm{C} 1-\mathrm{Cd} 1-\mathrm{O} 2-\mathrm{C} 1 \\
& \mathrm{Cd} 1-\mathrm{O} 2-\mathrm{C} 1-\mathrm{O} 1 \\
& \mathrm{Cd} 1-\mathrm{O} 2-\mathrm{C} 1-\mathrm{C} 2 \\
& \mathrm{Cd} 1-\mathrm{O} 1-\mathrm{C} 1-\mathrm{O} 2 \\
& \mathrm{Cd} 1-\mathrm{O} 1-\mathrm{C} 1-\mathrm{C} 2 \\
& \mathrm{O} 2-\mathrm{C} 1-\mathrm{C} 2-\mathrm{C} 7 \\
& \mathrm{O} 1-\mathrm{C} 1-\mathrm{C} 2-\mathrm{C} 7
\end{aligned}
$$

$126.84(3)$

$92.21(3)$

$54.80(3)$

$131.59(5)$

$114.36(4)$

$115.02(4)$

$95.34(4)$

$27.48(4)$

113.08 (4)

$27.33(4)$

$119.99(9)$

$107.90(6)$

$108.43(6)$

$108.43(6)$

$107.90(6)$

$102.86(8)$

$-130.73(7)$

$-26.66(10)$

$86.14(8)$

$-0.88(7)$

$140.90(7)$

$113.69(8)$

$72.63(8)$

$164.60(7)$

$-97.38(8)$

$0.89(7)$

$-54.85(7)$

$-78.93(9)$

$-1.48(11)$

$178.02(11)$

$1.67(13)$

$-177.85(9)$

$-24.41(18)$

$155.11(11)$
$\mathrm{C} 4-\mathrm{C} 3-\mathrm{C} 2$

$\mathrm{C} 4-\mathrm{C} 3-\mathrm{H} 3$

$\mathrm{C} 2-\mathrm{C} 3-\mathrm{H} 3$

$\mathrm{C} 5-\mathrm{C} 4-\mathrm{C} 3$

$\mathrm{C} 5-\mathrm{C} 4-\mathrm{H} 4$

$\mathrm{C} 3-\mathrm{C} 4-\mathrm{H} 4$

$\mathrm{C} 4-\mathrm{C} 5-\mathrm{C} 6$

$\mathrm{C} 4-\mathrm{C} 5-\mathrm{S} 1$

$\mathrm{C} 6-\mathrm{C} 5-\mathrm{S} 1$

$\mathrm{C} 7-\mathrm{C} 6-\mathrm{C} 5$

$\mathrm{C} 7-\mathrm{C} 6-\mathrm{H} 6$

$\mathrm{C} 5-\mathrm{C} 6-\mathrm{H} 6$

$\mathrm{C} 6-\mathrm{C} 7-\mathrm{C} 2$

$\mathrm{C} 6-\mathrm{C} 7-\mathrm{H} 7$

$\mathrm{C} 2-\mathrm{C} 7-\mathrm{H} 7$

$\mathrm{O} 2-\mathrm{C} 1-\mathrm{C} 2-\mathrm{C} 3$

$\mathrm{O} 1-\mathrm{C} 1-\mathrm{C} 2-\mathrm{C} 3$

$\mathrm{C} 7-\mathrm{C} 2-\mathrm{C} 3-\mathrm{C} 4$

$\mathrm{C} 1-\mathrm{C} 2-\mathrm{C} 3-\mathrm{C} 4$

$\mathrm{C} 2-\mathrm{C} 3-\mathrm{C} 4-\mathrm{C} 5$

$\mathrm{C} 3-\mathrm{C} 4-\mathrm{C} 5-\mathrm{C} 6$

$\mathrm{C} 3-\mathrm{C} 4-\mathrm{C} 5-\mathrm{S} 1$

$\mathrm{O} 3{ }^{\mathrm{ii}}-\mathrm{S} 1-\mathrm{C} 5-\mathrm{C} 4$

$\mathrm{O} 3-\mathrm{S} 1-\mathrm{C} 5-\mathrm{C} 4$

$\mathrm{C} 5$ ii- $1-\mathrm{C} 5-\mathrm{C} 4$

$\mathrm{O} 3{ }^{\mathrm{ii}}-\mathrm{S} 1-\mathrm{C} 5-\mathrm{C} 6$

$\mathrm{O} 3-\mathrm{S} 1-\mathrm{C} 5-\mathrm{C} 6$

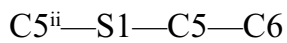

$\mathrm{C} 4-\mathrm{C} 5-\mathrm{C} 6-\mathrm{C} 7$

$\mathrm{S} 1-\mathrm{C} 5-\mathrm{C} 6-\mathrm{C} 7$

$\mathrm{C} 5-\mathrm{C} 6-\mathrm{C} 7-\mathrm{C} 2$

$\mathrm{C} 3-\mathrm{C} 2-\mathrm{C} 7-\mathrm{C} 6$

$\mathrm{C} 1-\mathrm{C} 2-\mathrm{C} 7-\mathrm{C} 6$
$119.75(13)$

120.1

120.1

119.95 (13)

120.0

120.0

120.73 (11)

119.41 (11)

$119.84(10)$

$119.16(12)$

120.4

120.4

120.54 (13)

119.7

119.7

$156.57(12)$

$-23.92(16)$

$2.62(18)$

$-178.34(11)$

$-1.87(19)$

$0.49(19)$

$178.80(10)$

$36.48(11)$

$167.88(10)$

$-77.63(10)$

$-145.19(10)$

$-13.78(12)$

$100.71(11)$

$0.15(19)$

$-178.16(10)$

$0.61(18)$

$-2.00(18)$

$178.99(11)$

Symmetry codes: (i) $-x, y,-z+3 / 2$; (ii) $-x+1, y,-z+5 / 2$.

Hydrogen-bond geometry $\left(A,{ }^{\circ}\right)$

\begin{tabular}{lllll}
\hline$D-\mathrm{H} \cdots A$ & $D-\mathrm{H}$ & $\mathrm{H} \cdots A$ & $D \cdots A$ & $D-\mathrm{H}^{\cdots} A$ \\
\hline $\mathrm{O} 4-\mathrm{H} 4 B \cdots \mathrm{O} 1^{\mathrm{iii}}$ & 0.85 & 1.97 & $2.7479(14)$ & 151 \\
$\mathrm{O} 4-\mathrm{H} 4 A \cdots \mathrm{O} 2^{\text {iv }}$ & 0.85 & 2.00 & $2.7364(15)$ & 145 \\
$\mathrm{C} 6-\mathrm{H} 6 \cdots \mathrm{O} 3$ & 0.93 & 2.55 & $2.9208(16)$ & 104 \\
\hline
\end{tabular}

Symmetry codes: (iii) $x, y-1, z$; (iv) $x,-y, z-1 / 2$. 